\title{
An Approximate Ablative Thermal Protection System Sizing Tool for Entry System Design
}

\author{
John A. Dec* \\ NASA Langley Research Center, Hampton, Virginia, 23681 \\ Robert D. Braun ${ }^{\dagger}$ \\ Georgia Institute of Technology, Atlanta, Georgia, 30332-0150
}

\begin{abstract}
A computer tool to perform entry vehicle ablative thermal protection systems sizing has been developed. Two options for calculating the thermal response are incorporated into the tool. One, an industry-standard, high-fidelity ablation and thermal response program was integrated into the tool, making use of simulated trajectory data to calculate its boundary conditions at the ablating surface. Second, an approximate method that uses heat of ablation data to estimate heat shield recession during entry has been coupled to a one-dimensional finite-difference calculation that calculates the in-depth thermal response. The in-depth solution accounts for material decomposition, but does not account for pyrolysis gas energy absorption through the material. Engineering correlations are used to estimate stagnationpoint convective and radiative heating as a function of time. The sizing tool calculates recovery enthalpy, wall enthalpy, surface pressure, and heat transfer coefficient. Verification of this tool is performed by comparison to past thermal protection system sizings for the Mars Pathfinder and Stardust entry systems and calculations are performed for an Apollo capsule entering the atmosphere at lunar and Mars return speeds.
\end{abstract}

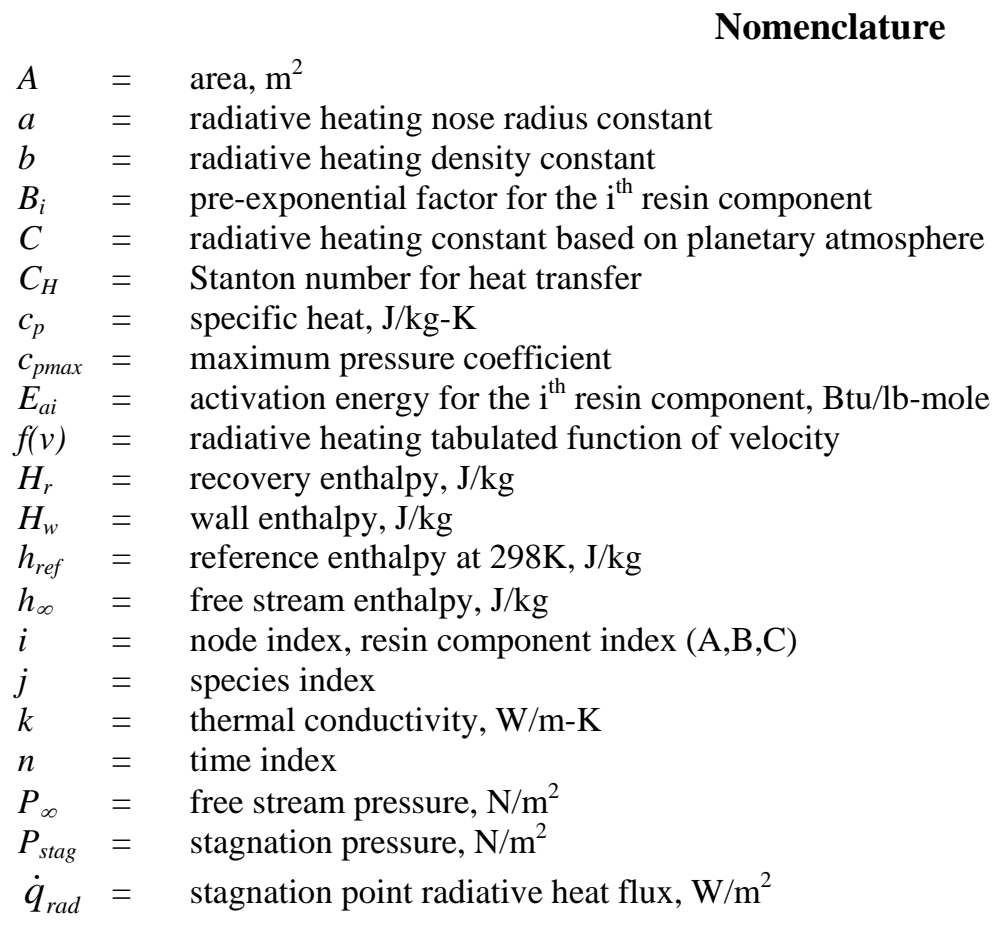

\footnotetext{
*AIAA Member, Aerospace Engineer, Structural and Thermal Systems Branch, MS 431

${ }^{\dagger}$ AIAA Associate Fellow, David and Andrew Lewis Associate Professor of Space Technology, Guggenheim School of Aerospace Engineering
} 


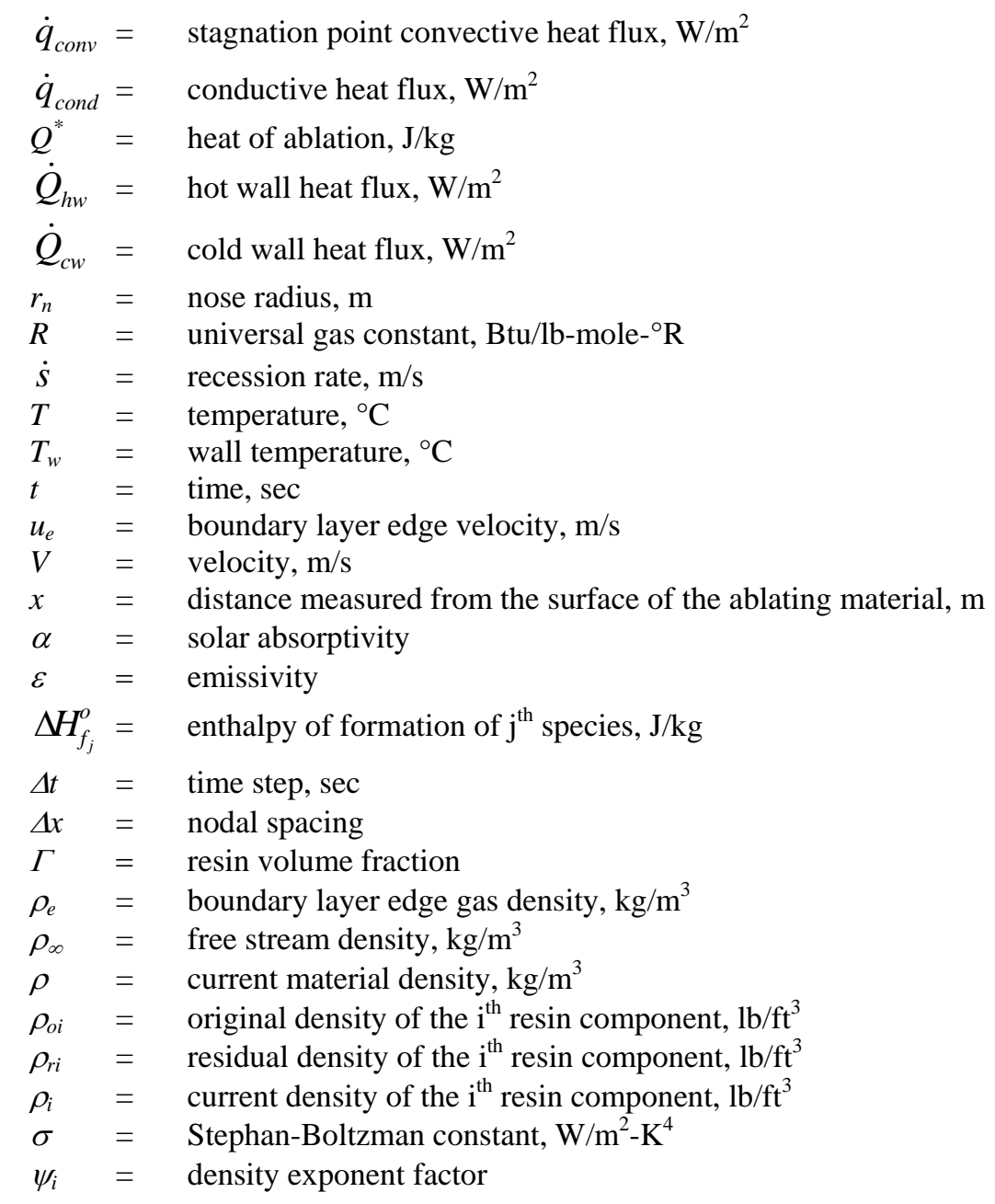

\section{Introduction}

CALCULATING the thermal response of an entry vehicle's thermal protection system (TPS) can be quite complex. A high-fidelity calculation of the thermal response using a program such as Aerotherm's Charring Material Thermal Response and Ablation Program ${ }^{1}$, or CMA, can be quite involved, particularly when one considers the enormous amount of data required as input by the program. In particular, generating the surface chemistry input and the pyrolysis gas enthalpy, requires that an additional equilibrium, or non-equilibrium chemistry code be run. These chemistry codes have extensive input of their own and require knowledge of the composition of the material as well as the composition of the pyrolysis gas. To complete this analysis, a thermal response model must be developed and correlated to arc jet test data, a set of surface chemistry tables that encompass a wide range of surface conditions must be available, and a pyrolysis gas enthalpy model must be developed. If these models do not exist and the exact composition of the pyrolysis gas and the TPS material are not known, constructing and running a highfidelity CMA model is problematic from both an accuracy and solution convergence standpoint.

Less complex methods for calculating the thermal response of a TPS material are useful when a high-fidelity thermal response model is not available, particularly during conceptual design where a large number of rapid trade studies are to be performed. These approximate methods generally employ the steady state ablation assumption and use the heat of ablation, or $\mathrm{Q}^{*}$, to predict the amount of recession during entry ${ }^{2}$. These methods often use an approximate analytic equation to determine the transient one-dimensional heat conduction through the material. The approximate heat conduction equation is used to solve for the required insulation thickness given a pre-defined TPSstructure interface (bondline) temperature. These methods provide the systems designer with a "ball-park" answer, but are lacking in that the steady state ablation assumption is usually not valid during flight and will generally overpredict recession. Such methods also lack in that the approximate transient heat conduction equations used are 
typically only valid where either semi-infinite solid or planar-wall assumptions hold ${ }^{3}$. These heat conduction approximations do not account for material decomposition, where the density, thermal conductivity, and specific heat change with both temperature and as the material pyrolyizes and goes from a virgin to a charred composite. They also do not account for the pyrolysis gas percolating through the material and absorbing energy.

The main goal of the current TPS sizing tool is to provide an improved approximate method suitable for conceptual design, when a high fidelity thermal response model for the material does not exist. In addition, this investigation provides a means by which a high-fidelity thermal response calculation can be performed with little difficulty when a thermal response model does exist. The tool accomplishes this by using trajectory input from a source such as the Program to Optimize Simulated Trajectories (POST) ${ }^{4}$, uses vehicle geometric data defined by the user, uses engineering correlations to estimate stagnation-point convective and radiative heating, and performs an iterative one-dimensional thermal response calculation to size the thermal protection system for a wide range of vehicle sizes, shapes, and TPS materials. Since a one-dimensional thermal response calculation is performed, this tool is generally applicable at the heatshield stagnation point only. Two options for calculating thermal response are incorporated into the tool. The first option is a complete thermal response calculation using CMA. The second is an approximate solution using the heat of ablation to predict recession. Instead of an approximate analytic solution for the transient heat conduction through the material, a transient, one-dimensional finite-difference heat transfer calculation is used for the in-depth thermal response. This finite-difference formulation accounts for material decomposition by allowing the density, thermal conductivity, and specific heat to vary with temperature and material char state. It also provides a way to extract the in-depth temperature history behind the stagnation point. The finite-difference solution does not include pyrolysis gas energy absorption through the material since this would require the pyrolysis gas enthalpy. If a model for the pyrolysis enthalpy exists, one would be better off running the complete CMA solution (option 1).

\section{Approach}

In general, TPS sizing is performed by reading input trajectory data from POST and other user-defined input, determining the necessary boundary conditions for the thermal response calculation, and iteratively sizing the heat shield thickness to satisfy the user-defined bondline temperature criteria at the stagnation point. Given the vehicle geometry and the TPS material density, the total mass of the heatshield can be calculated, assuming uniform thickness over the entire surface of the vehicle's forebody. The TPS mass can then be fed back into POST and the trajectory recalculated based on this new mass estimate. Iteration between POST and the TPS sizing continues until convergence is reached. Integration of the relevant disciplinary analyses including aeroshell geometry, atmospheric modeling, vehicle aerodynamics, atmospheric flight mechanics, aerothermodynamics, and thermal analysis within a single multi-disciplinary framework has been accomplished in the Planetary Entry Systems Synthesis Tool, or PESST $^{5}$.

\section{A. Reading Trajectory and Vehicle Data}

Trajectory output data is simply stored into arrays for use by the TPS sizing tool. A separate input file containing user-defined input contains vehicle information including TPS material type, nose radius, an initial estimate of TPS thickness and the initial temperature of the TPS.

\section{B. Material Properties}

In order to remove some of the complexity involved in running a thermal response calculation, a database was constructed that gives the user access to five common ablative materials and eight materials commonly used in the substructure of the aeroshell. The database is flexible in that materials can be easily added without having to modify the TPS tool source code. The format of the data contained in the database falls into two main categories: thermophysical property data and surface chemistry data.

The thermophysical data includes both ablative and substructure material properties. Ablative material thermophysical properties include decomposition kinetic constants used in the Arrhenius formulation for density decomposition, resin volume fraction, heats of formation, thermal conductivity, specific heat, emissivity, pyrolysis gas enthalpy, and heat of ablation curve fit constants. The thermal conductivity, specific heat, and emissivity are input as functions of temperature and have property entries for both virgin and char material. The pyrolysis gas enthalpy is a function of temperature and pressure. Substructure material properties are similar to the ablative material properties with the following exceptions: (a) instead of the decomposition kinetic constants, only the material density is input; (b) there are no entries for the resin volume fraction and heats of formation; (c) there is only one entry for the specific heat and thermal conductivity as a function of temperature (since there is no 
distinction between virgin and char for the substructure); and (d) there is also no need for the pyrolysis gas enthalpy. Table 1 lists the materials available. The surface chemistry data file and pyrolysis gas enthalpy are only required for the CMA solution and are only required for an ablative material. The surface chemistry data is very specific and need not be modified. As such, a detailed description of it is not presented here. The interested reader is referred to Ref. 1 for a complete description of the surface chemistry data.

Table 1. Material database

\begin{tabular}{ccc}
\hline \hline Material Name & Description & Ablator \\
\hline Carbon-Carbon & T-300 Carbon-Carbon & Yes/No \\
FM5055 & High-density carbon phenolic & Yes \\
FM5504 & Silica phenolic & Yes \\
P50 Cork & P50 Cork, launch vehicle grade & Yes \\
PICA & Mid-density, phenolic impregnated ceramic ablator & Yes \\
SLA-561V & Low-density, cork-silicone & Yes \\
Aluminum & Generic 2024 aluminum alloy & No \\
AL HC 1.6pcf & Aluminum honeycomb 1.6 lbs per ft & No \\
Copper & Generic Copper & No \\
EAC & Energy absorbing foam material & No \\
Equiv. MLI & Equivalent conductor for multi-layer insulation & No \\
Fiber Frax & Carbon based insulator & No \\
GR Epxy & Generic graphite epoxy & No \\
RTV-560 & Adhesive & No \\
\hline \hline
\end{tabular}

\section{Stagnation-Point Heating}

Engineering correlations are used to estimate the stagnation-point convective and radiative heat rate. Each of these correlations assumes a cold-wall boundary condition. The Sutton-Graves ${ }^{6}$ convective heating relation is used and is given as:

$$
\dot{q}_{c o n v}=k \cdot\left(\frac{\rho}{r_{n}}\right)^{1 / 2} \cdot V^{3}
$$

The constant in this equation is a function of atmospheric chemistry, where the following constants are used for Earth and Mars:

$$
\begin{array}{ll}
k=1.74153 \times 10^{-4} & \text { (For Earth) } \\
k=1.9027 \times 10^{-4} \quad \text { (For Mars) }
\end{array}
$$

This stagnation-point engineering correlation has been verified against numerous ground-based test and flight programs and is generally accurate for blunt bodies to within 5-10\%.

Stagnation-point radiative heating is calculated using the Tauber-Sutton correlation for Earth and Mars ${ }^{7}$. The Tauber-Sutton formulation is given as:

$$
\dot{q}_{\text {rad }}=C \cdot r_{n}^{a} \cdot \rho_{\infty}^{b} f(V)
$$

Equation 2 gives the radiative heat rate in $\mathrm{W} / \mathrm{cm}^{2}$. The constants a and $\mathrm{b}$ depend on the velocity, density, planetary atmosphere, and vehicle nose radius and are defined in Ref. 7. The function $f(V)$ is a tabulated function of velocity and is also given in Ref. 7. For Earth, the above formulation is generally valid for entry vehicle nose radii between 0.3 and 3.0 meters, velocities between 10 and $16 \mathrm{~km} / \mathrm{s}$, and altitudes between 72 and $54 \mathrm{~km}$. For Mars, the above formulation is for valid for entry vehicle nose radii between 1.0 and 23.0 meters, velocities between 6.5 and $9 \mathrm{~km} / \mathrm{s}$, and between 51 and $30 \mathrm{~km}$ altitudes. Tauber and Sutton demonstrated that if the above expression is used outside its range of applicability, the resulting error is still generally within the range of uncertainty found in computing the radiative heating with a more computationally intense method. 
Stagnation-point convective and radiative heat rates are added to produce the total heat rate at each point along the trajectory. The total radiative heat load is calculated by integrating the radiative heating over the trajectory and then is added to the total convective heat load. The radiative heat rate, convective heat rate, and total heat load are output as a function of time.

\section{Integration with CMA}

The first option for calculating the thermal response is to run CMA. The CMA executable is linked to the main program as a dynamic link library. The interface between CMA and the main program is the same as if it were a subroutine; the only difference is that if changes are made to the main program, the CMA portion does not need to be recompiled in order for the code to run. The main benefit of coupling CMA directly to the main program, and in effect, to POST, is that the boundary conditions necessary to run CMA are automatically calculated and the CMA input files are automatically generated by the main program. Along with the material database, this allows for the performance of multiple CMA runs without having to recalculate the boundary conditions or update the CMA input.

The boundary conditions required by CMA as a function of time are calculated from the trajectory data. CMA is enthalpy based and uses the recovery enthalpy and a heat transfer coefficient to compute the convective heat flux. The stagnation-point recovery enthalpy is calculated using equation 3.

$$
H_{r}=\frac{V^{2}}{2}+h_{\infty}-h_{r e f}+\sum \Delta H_{f_{j}}^{0}
$$

The free stream enthalpy for air is calculated using TGAS, a program that calculates the equilibrium properties of air based on curve fits ${ }^{8}$. TGAS is linked to the main program as a dynamic link library just like the CMA module. For Earth, the enthalpy of formation for $\mathrm{N}_{2}$ and $\mathrm{O}_{2}$ are 0.0 ; for Mars, the $\mathrm{CO}_{2}$ enthalpy of formation is approximately $-8942.0 \mathrm{~kJ} / \mathrm{kg}$. With the recovery enthalpy, and the cold wall heat rate, the heat transfer coefficient can be calculated as shown in equation 4.

$$
\rho_{e} u_{e} C_{H}=\frac{\dot{Q}_{h w}}{\left(H_{r}-H_{w}\right)}
$$

The cold wall heat flux can be substituted for the hot wall heat flux in this equation for more conservative results. The hot wall heat flux is related to the cold wall heat flux by equation 5 .

$$
\dot{Q}_{h w}=\dot{Q}_{c w}\left(1-\frac{H_{w}}{H_{r}}\right)
$$

CMA also requires the surface pressure incident on the vehicle. The surface pressure is calculated using Modified Newtonian Theory, where the pressure at the stagnation point is given by equation 6 .

$$
P_{\text {stag }}=P_{\infty}+\frac{1}{2} \rho_{\infty} V^{2} C_{p_{\max }}
$$

The process of calculating the recovery enthalpy, heat transfer coefficient, and stagnation-point pressure is performed at every time point in the trajectory. Computational efficiency is improved by avoiding large boundary condition files. If the array of boundary conditions has more than 50 time points, it is condensed to 50 points and written to the CMA boundary condition file. The first 40 points selected are centered around peak heating starting at time, $\mathrm{t}=0$ seconds, and are equally spaced before and after peak heating. The last 10 points continue where the 40th point left off and are equally spaced up to the last time entry. 


\section{E. Approximate Heat of Ablation and Finite-Difference Calculation}

The second option for calculating thermal response of the TPS is to use an approximate technique. There are two components to the approximate technique presented here. The first component makes use of the steady state ablation assumption and employs the heat of ablation, $Q^{*}$, to estimate the recession during entry. The second component involves calculating the in-depth temperature response to estimate the amount of material required as insulation to keep the bondline temperature below a specified limit. Calculating the in-depth temperature response is accomplished using a finite-difference formulation for the in-depth conduction through the material.

Using the heat of ablation, the recession rate at any instant in time can be calculated by equation 7 . The total recession is then found by integrating the recession rate over the trajectory. This formulation is only valid for steadystate ablation; whereas, in the transient aerothermodynamic environment of atmospheric entry, steady-state ablation is difficult to achieve.

$$
\dot{s}=\frac{\dot{Q}_{h w}}{\rho \cdot Q^{*}}
$$

That being said, equation 7 is conservative and will generally over predict the actual recession rate. This over prediction will grow as material density decreases and the heat rate increases. To correct for this over prediction, a recession threshold temperature was incorporated, below which the recession rate is set to zero. For example, it is known that little recession will occur for carbon phenolic in air when the surface temperature is below $2000 \mathrm{~K}$. Using $2000 \mathrm{~K}$ as the recession threshold temperature will ensure that recession is only calculated when the surface temperature exceeds $2000 \mathrm{~K}$, thus minimizing error. Note that the recession estimate using the recession threshold temperature and equation 7 is compared to option 1 calculations using CMA in the results section of this document.

The one-dimensional heat conduction equation can be written along with the surface energy balance as shown in Eqs. 8a and 8b.

$$
\begin{gathered}
\rho c_{p} \frac{\partial T}{\partial t}=\frac{1}{A} \frac{\partial}{\partial x}\left(k A \frac{\partial T}{\partial x}\right) \\
\dot{q}_{\text {conv }}+\alpha \dot{q}_{\text {rad }}-\dot{q}_{\text {cond }}-\varepsilon \sigma T_{w}^{4}=0
\end{gathered}
$$

This formulation neglects various forms of chemical fluxes entering the surface as well as the pyrolysis energy rate and the net energy absorbed due to pyrolysis gas movement through the material in the in-depth solution. The material decomposition, or the change in density, is computed explicitly as if it were a material property. The change in density as a function of temperature is computed using the same Arrhenius equation used by CMA, which is shown in Eq. 9. This formulation uses a three-component model for the density decomposition. All of the parameters for Eq. 9 are contained in the thermophysical material property data previously described.

$$
\frac{\partial \rho_{i}}{\partial t}=-\mathrm{B}_{i} \exp \left(\frac{-E_{a_{i}}}{R T}\right) \rho_{o_{i}}\left(\frac{\rho_{i}-\rho_{r_{i}}}{\rho_{o_{i}}}\right)^{\psi_{i}}
$$

The instantaneous density for the composite is given by Eq. 10 .

$$
\rho=\Gamma\left(\rho_{A}+\rho_{B}\right)+(1-\Gamma) \rho_{C}
$$

The implicit discretization of the one-dimensional heat conduction equation is performed using a finite-volume or control-volume technique ${ }^{9}$. The derivation is somewhat lengthy and will not be reproduced here. The results of the discretization for the node at the surface and any interior node are shown in Eqs. 11a and 11b, respectively. 


$$
\begin{gathered}
\left(1+\frac{2 k \Delta t}{\rho c_{p} \Delta x_{i}^{2}}\right) T_{i}^{n+1}-\frac{2 k \Delta t}{\rho c_{p} \Delta x_{i}^{2}} T_{i+1}^{n+1}=T_{i}^{n}+\dot{q}_{\text {rad }} \frac{2 \Delta t}{\rho c_{p} \Delta x_{i}}+\dot{q}_{\text {conv }} \frac{2 \Delta t}{\rho c_{p} \Delta x_{i}}-\varepsilon \sigma T_{i}^{n^{4}} \frac{2 \Delta t}{\rho c_{p} \Delta x_{i}} \\
T_{i}^{n}=-\frac{k \Delta t}{\rho c_{p} \Delta x_{i}^{2}} T_{i-1}^{n+1}+\left(1+\frac{2 k \Delta t}{\rho c_{p} \Delta x_{i}^{2}}\right) T_{i}^{n+1}-\frac{k \Delta t}{\rho c_{p} \Delta x_{i}^{2}} T_{i+1}^{n+1}
\end{gathered}
$$

Equations 11a and 11b can be assembled into a banded, tri-diagonal matrix and solved using the Thomas algorithm for the nodal temperatures ${ }^{10}$. As mentioned previously, density is evaluated explicitly using Eqs. 9 and 10. This explicit evaluation can introduce instability to the solution and requires that small time steps or node sizes be used.

\section{F. Finite-Difference Grid Generation and Iterative TPS Sizing}

Both the CMA and approximate solution require a finite-difference grid. The finite-difference grid for the approximate solution uses a uniform grid. A simple algorithm is implemented which takes the users initial estimate of TPS thickness and the desired maximum nodal spacing, $\Delta \mathrm{x}$, and computes the number of nodes required to keep the nodal spacing just below or equal to the maximum desired spacing. The nodes are then numbered and stored in an array along with a material number flag to keep track of each node's material. CMA uses a forward biased grid, where the node size decreases as the surface is approached. This is implemented by pre-defining the first fifteen nodes, then using the same uniform grid (as in the approximate solution) for the rest of the thickness. The first fifteen pre-defined nodal spacings are based on previous experience and work for a wide range of materials and boundary conditions ${ }^{1}$.

The iteration scheme to size the TPS given the bondline temperature criteria is the same for both the CMA solution and the approximate solution. The secant method ${ }^{10}$ is used and converges without difficulty within 10-15 iterations. Although a Newton iteration scheme would likely converge in fewer iterations, this scheme was not chosen for this particular application because an accurate calculation of the derivative and a very good guess of the initial TPS thickness would be required to guarantee convergence. The procedure for finding the TPS thickness given a bondline temperature criteria and a trajectory are summarized as follows. The first step is to perform a thermal response calculation using the initial estimate of the TPS thickness. As required by the secant method, a second calculation is performed at a thickness $0.1 \mathrm{~cm}$ thicker than the initial estimate. If there is not a significant difference in the bondline temperature between these two calculations, the initial estimate of the TPS thickness is cut in half and the calculations are repeated. Once these initial calculations are complete, the secant method methodically adjusts the TPS thickness until the calculated bondline temperature matches the given bondline temperature to within $0.1^{\circ} \mathrm{C}$.

\section{Verification}

To verify the calculations of the CMA and the approximate thermal response options, entry trajectories for two flight systems were examined: the Mars Pathfinder and Stardust entry systems. Mars Pathfinder, a Mars entry vehicle had a $0.6625 \mathrm{~m}$ nose radius, ballistic coefficient of $63 \mathrm{~kg} / \mathrm{m}^{2}$, inertial entry velocity of $7.26 \mathrm{~km} / \mathrm{s}$, and a SLA$561 \mathrm{~V}$ forebody TPS ${ }^{11}$. The estimated peak convective stagnation-point heat rate for Mars Pathfinder was around 106 $\mathrm{W} / \mathrm{cm}^{2}$, with a $3865 \mathrm{~J} / \mathrm{cm}^{2}$ total heat $\operatorname{load}^{11}$. In this study, the peak heat rate used was slightly higher than encountered in flight, with a combined convective and radiative heat rate of $119 \mathrm{~W} / \mathrm{cm}^{2}$ and total heat load of 3880 $\mathrm{J} / \mathrm{cm}^{2}$. Figure 1 shows the convective and radiative stagnation-point heat rates extracted from Milos and $\mathrm{Chen}^{12}$ and is plotted along with the calculated convective and radiative stagnation-point heat rate and calculated total stagnation-point heat load. Figure 1 shows that the peak heat rate and total heat load used in this study are similar to the values used by Milos and $\mathrm{Chen}^{12}$, in this case the combined convective and radiative peak heat rate is around 120 $\mathrm{W} / \mathrm{cm}^{2}$ and the total heat load is $3800 \mathrm{~J} / \mathrm{cm}^{2}$. From Milos and $\mathrm{Chen}^{12}$, the calculated thickness of the Mars Pathfinder heat shield for $120 \mathrm{~W} / \mathrm{cm}^{2}$ peak heat rate case was $1.4 \mathrm{~cm}$ without margin. To account for uncertainty, margin was added which increased the TPS thickness to $1.9 \mathrm{~cm}$. Using the current TPS sizing tool, the "no margin" heat shield thickness was calculated to be $1.57 \mathrm{~cm}$ with the approximate technique. CMA calculated a $0.91 \mathrm{~cm}$ "no margin" TPS thickness. While this appears to be a good correlation for the approximate technique, the CMA solution under predicts the thickness obtained by Milos and Chen by $0.49 \mathrm{~cm}$. The surface and bondline temperature histories found 


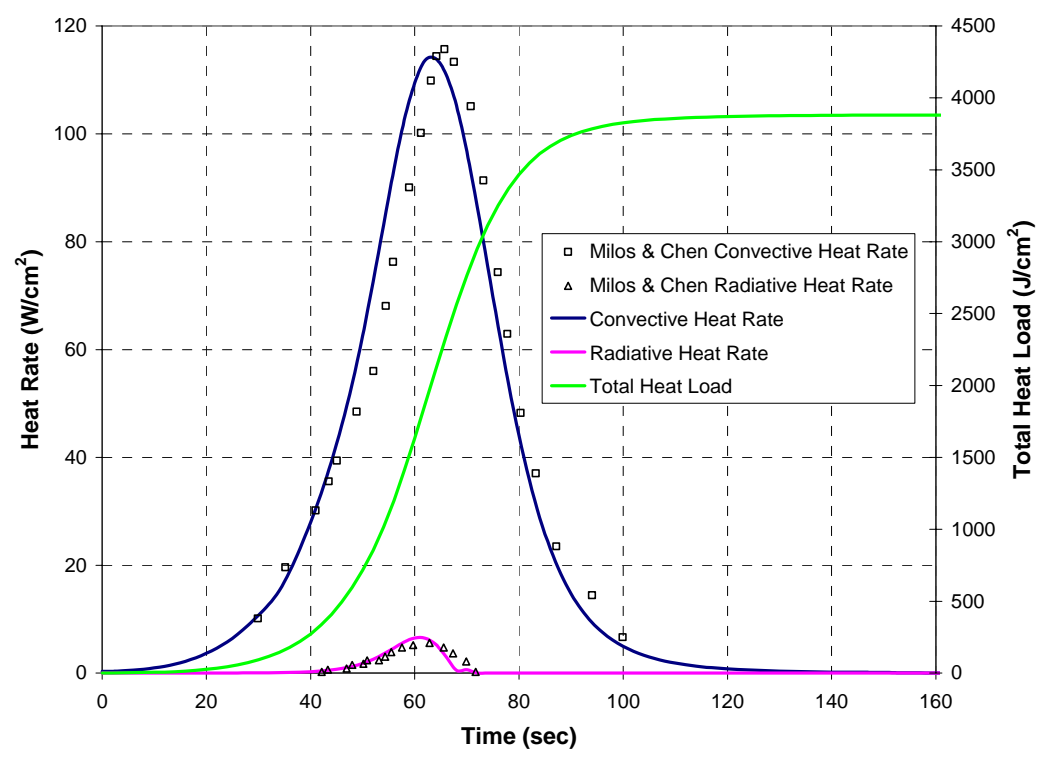

Fig. 1 Mars Pathfinder heat rate and total heat load

in Milos and Chen were calculated using the margined TPS thickness of $1.9 \mathrm{~cm}$. The data was presented for the margined case since Milos and Chen were comparing their calculations to flight data. In order to compare the surface and bondline temperatures calculated in this study with Milos and Chen, it was necessary to make additional calculations using both CMA and the approximate tool with a TPS thickness of $1.9 \mathrm{~cm}$ using the same trajectory and heat rates. The surface and bondline temperature histories for the $1.9 \mathrm{~cm}$ thick case are shown in Figs. 2 and 3. Figure 2 shows the peak temperatures reached by each of the three methods differ slightly in magnitude. In particular, the peak stagnation point surface temperature prediction from the CMA solution shows that the surface only reaches $1607^{\circ} \mathrm{C}$ at $60 \mathrm{sec}$ into the trajectory. Calculations performed by Milos and Chen ${ }^{12}$ using the Fully Implicit Ablation and Thermal Response program, or FIAT, show that the Mars Pathfinder TPS reaches a peak stagnation point temperature of about $1760^{\circ} \mathrm{C}$ at approximately $64 \mathrm{sec}$ into the trajectory (slightly after the peak heat rate condition). The approximate solution shows the surface temperature peaking at $1865^{\circ} \mathrm{C}$ just after peak heat rate condition at $64 \mathrm{sec}$.

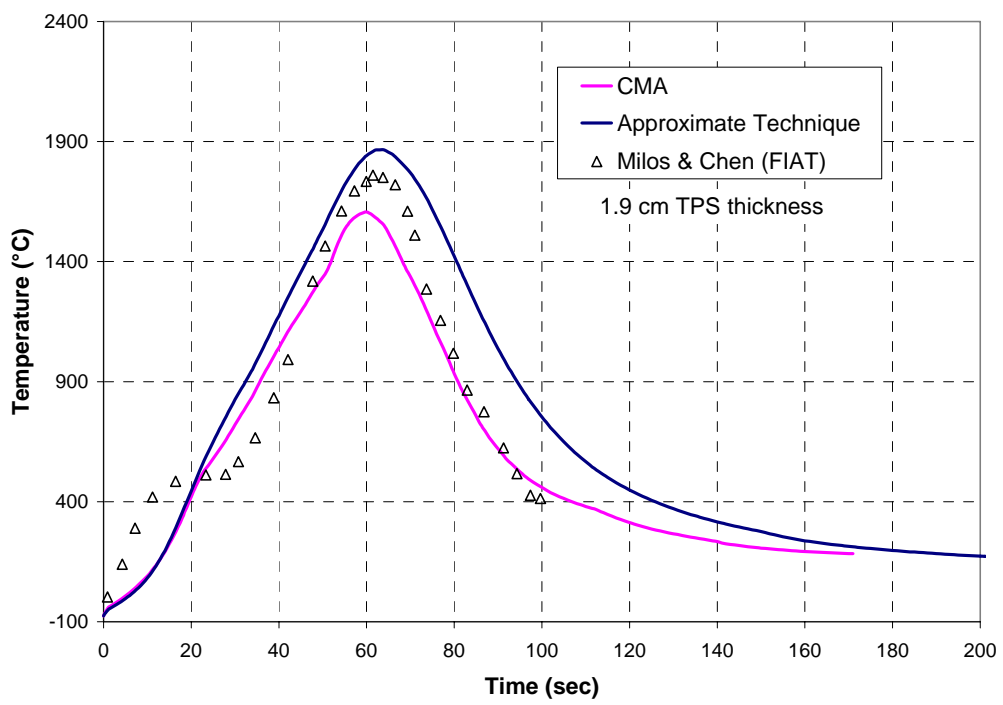

Fig. 2 Mars Pathfinder surface temperature comparison 
Referring back to the nominal sizing calculations, it was found that the surface temperatures obtained for the approximate and CMA solutions for the nominal sizing case were essentially equal to the $1.9 \mathrm{~cm}$ case, since the surface temperature is mostly a function of the peak heat rate and not effected as much by the overall thickness. Taking a closer look at the surface temperature prediction by CMA and examining the output of the solution more carefully explains how the predicted nominal thickness is so much lower than the nominal thickness calculated by Milos and Chen and calculated by the approximate tool. The output reveals the CMA model for SLA561-V is predicting a large amount of pyrolysis and charring of the material. The mass flux of the pryolysis gas and char are both inputs into the blowing correction made by CMA. The blowing correction is a factor used to reduce the heat transfer coefficient to account for the ablation products being injected into the boundary layer. The reduction in heat transfer coefficient in turn reduces the incident heat flux. A lower effective heat flux and hence a lower effective total heat load reduce the thickness required to maintain the bondline temperature within $250^{\circ} \mathrm{C}$. Also, the large amount of pyrolysis and charring in the material absorbs energy and further reduces the thickness requirement. In addition, this SLA561-V model predicted no recession, a small amount of recession would have also increased the calculated thickness. The FIAT code used by Milos and Chen is a derivative of CMA and given an identical model the two should produce similar results. The fact that the two solutions do not agree indicates the two models for SLA561-V do not match. This is most likely the case since the model used by Milos and Chen was correlated to specific arc jet data obtained for the Mars Pathfinder project and the SLA model used in this study was obtained from the material database TPSX (thermal protection system expert) ${ }^{13}$. It is likely that the pyrolysis gas enthalpy and surface thermochemistry data in this model are not correct for this particular atmosphere. Conversely, the approximate solution does not include the effects of pyrolysis or reactions at the surface and conservatively predicts a slightly higher surface temperature and nominal thickness.

Figure 3 shows that all three bondline temperature histories seem to match very well. This is a consequence of performing the calculations with a TPS thickness that is much thicker than required, and as shown, there is not much of a temperature increase. Since there is not much of a temperature increase in the region near the bondline, there is relatively little, if any, pyrolysis and charring of the material. Since the approximate solution does not account for blowing, and, or pyrolysis, Figure 3 shows that the approximate solution is calculating the in depth temperature response correctly, minus the small discrepancies due to pyrolysis and charring occurring closer to the surface in the FIAT and CMA solutions.

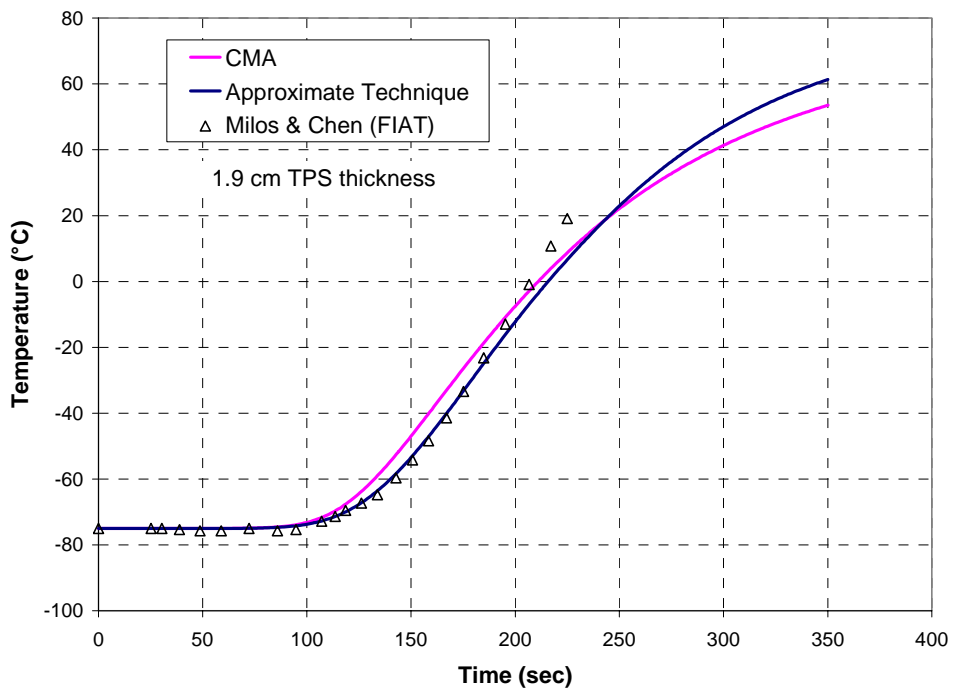

Fig. 3 Mars Pathfinder bondline temperature comparison

Atmospheric entry of the Stardust sample return capsule was also examined. Stardust is an Earth entry vehicle with a nose radius of $0.23 \mathrm{~m}$, ballistic coefficient of $60 \mathrm{~kg} / \mathrm{m}^{2}$, relative entry velocity of $12.6 \mathrm{~km} / \mathrm{s}$, and with a PICA forebody TPS ${ }^{14}$. The peak heat rate predicted for the Stardust sample return capsule is $1126 \mathrm{~W} / \mathrm{cm}^{2}$ with an estimated total heat load of $28,000 \mathrm{~J} / \mathrm{cm}^{2}$. For this entry condition the stagnation point TPS thickness was calculated to be $4.8 \mathrm{~cm}$ with $1.2 \mathrm{~cm}$ of recession ${ }^{15}$. The POST entry trajectory used for this Stardust verification is slightly different than that used to size the heatshield in the project's preliminary design phase. This trajectory has a lower peak stagnation-point heat rate and total stagnation-point heat load, has a slightly different timeline, and a slightly 
lower peak vehicle surface pressure. Figure 4 shows the stagnation-point convective and radiative heat rates plotted with the results from Olynick et. al. ${ }^{15}$ The heat rates from Oylnick, et al. could not be used directly because they were extracted from the reference using a digitizer software program, and unfortunately, other important trajectory information was absent, so they could not be placed in a POST output format for use in this tool. In order to make a viable comparison, the POST trajectory data available for Stardust was scaled to match the peak heat rate from

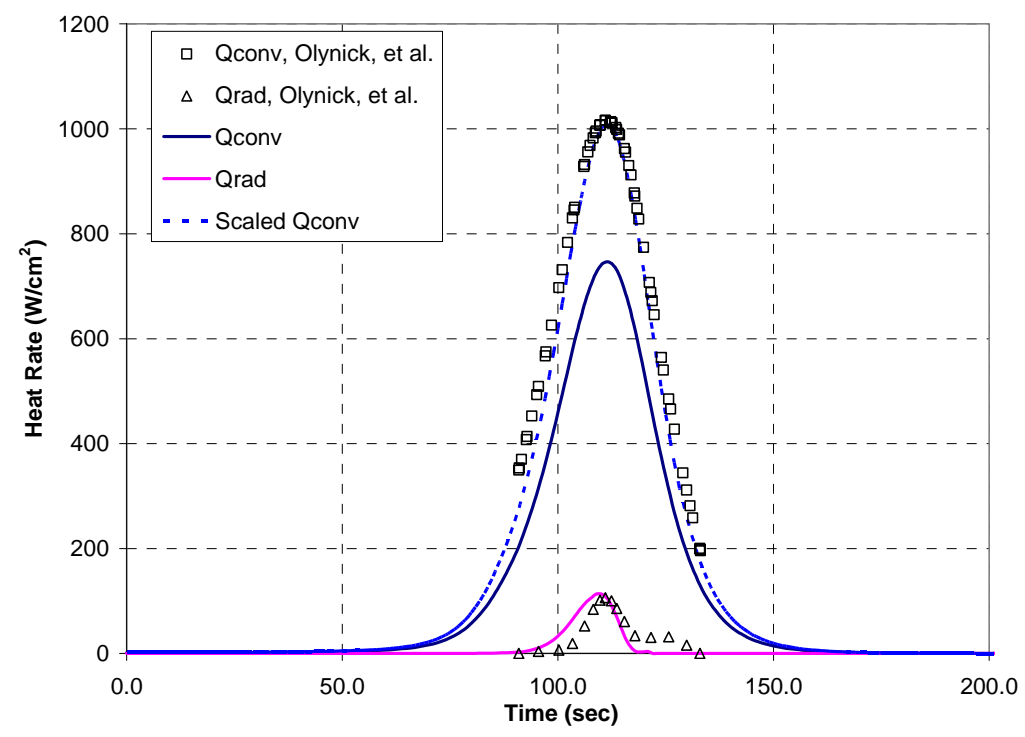

Fig. 4 Stardust heat rate

Olynick, et. al. and the time line of the Olynick data was shifted to match the POST trajectory. The peak pressure however, could not be matched. The combined convective and radiative peak heat rate for this scaled trajectory was $1126 \mathrm{~W} / \mathrm{cm}^{2}$ with a total heat load of about $28,800 \mathrm{~J} / \mathrm{cm}^{2}$. For the "scaled" entry case, the calculated TPS thickness using CMA was $4.52 \mathrm{~cm}$ with $1.12 \mathrm{~cm}$ of recession. The approximate technique produced a thickness of $4.78 \mathrm{~cm}$ with a recession of $0.726 \mathrm{~cm}$. These thicknesses are very close to the thickness calculated in reference 15, and it appears that the CMA and approximate solutions do a fairly good job at predicting the thermal response. Although the three solutions for thickness seem to correlate very well, there are a few minor discrepancies. The first discrepancy is the difference in surface temperature, shown in Figure 5, between the CMA solution and the approximate solution. As shown in Figure 5, Olynick computed two stagnation point surface temperatures, one with

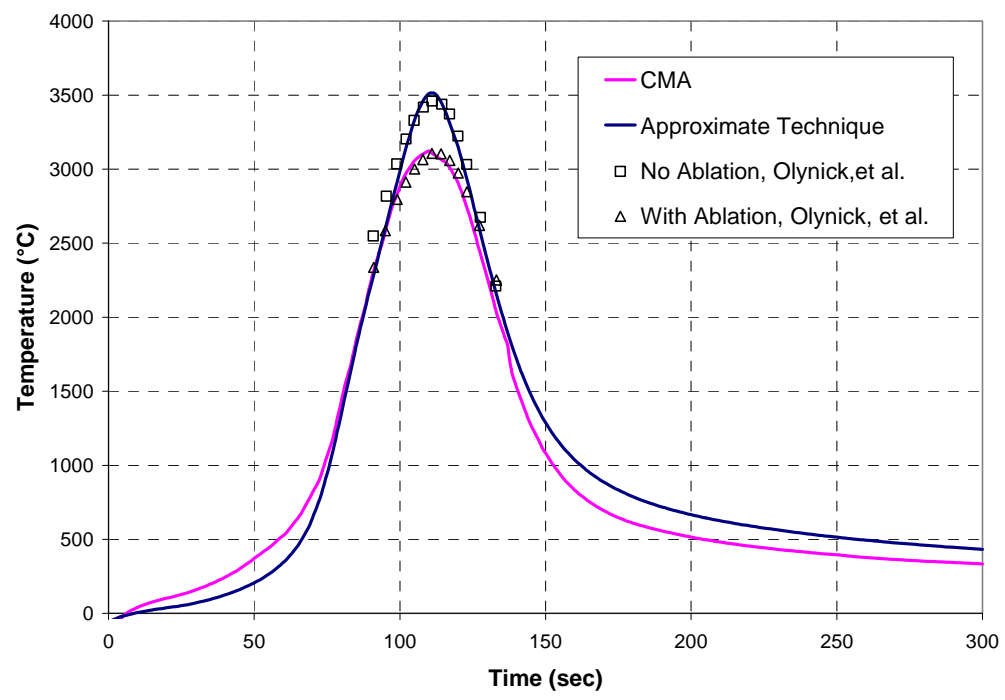

Fig. 5 Surface temperature comparison 
ablation the other with out the beneficial effect of ablation. The surface temperatures match the Olynick solutions very well, however, the approximate solution peaks at $3516^{\circ} \mathrm{C}$, about $395^{\circ} \mathrm{C}$ higher than the CMA solution which was $3121^{\circ} \mathrm{C}$. This occurrs because at this heat rate, the PICA heat shield is ablating at a high rate and injecting a large quantity of ablation products into the boundary layer which reduces the heat flux on the vehicles surface, and in turn lowers the surface temperature. Olynick et. al. show that the surface temperature for the non-ablating case peaks at $3460^{\circ} \mathrm{C}$ and the ablating case peaks at $3105^{\circ} \mathrm{C}$. Figure 5 illustrates that the approximate solution correlates very well to the case with no ablation, as would be expected, differing by $56^{\circ} \mathrm{C}$ at the peak. The CMA solution is shown to correlate very well to the case with ablation, differing by only $16^{\circ} \mathrm{C}$. Again, the discrepancy between the approximate solution and CMA can be explained by the fact that the approximate solution neglects the effects of pyrolysis gas energy absorption, pyrolysis gas convection through the solid and surface chemistry. The second discrepancy is the amount of recession predicted. The overall TPS thickness correlates very well, but the amount of recession predicted by the approximate method is $35 \%$ to $40 \%$ lower than the CMA and Olynick solutions. This is a consequence of using the heat of ablation to calculate the recession. Finally, if the amount of recession is subtracted from the calculated TPS thickness, what remains is the amount of insulation required to maintain the bondline below $250^{\circ} \mathrm{C}$. The amount of insulation required by CMA, the approximate, and Oylnick solutions are $3.4 \mathrm{~cm}, 4.054 \mathrm{~cm}$, and $3.6 \mathrm{~cm}$ respectively. The CMA and Olynick solutions match very well, the difference can be attributed to the model for the char properties of the PICA not being the same. The approximate method over predicts the insulation thickness by about $11 \%$ compared to Oylnick. Once again, the approximate technique does not account for the effects of pyrolysis energy absorption, pyrolysis gas convection through the solid and chemical reactions at the surface, all of which tend to lower the incoming heat flux and total heat load. Compared to CMA and Olynick, the approximate technique sees an overall higher heat flux and total heat load and thus requires more insulation to keep

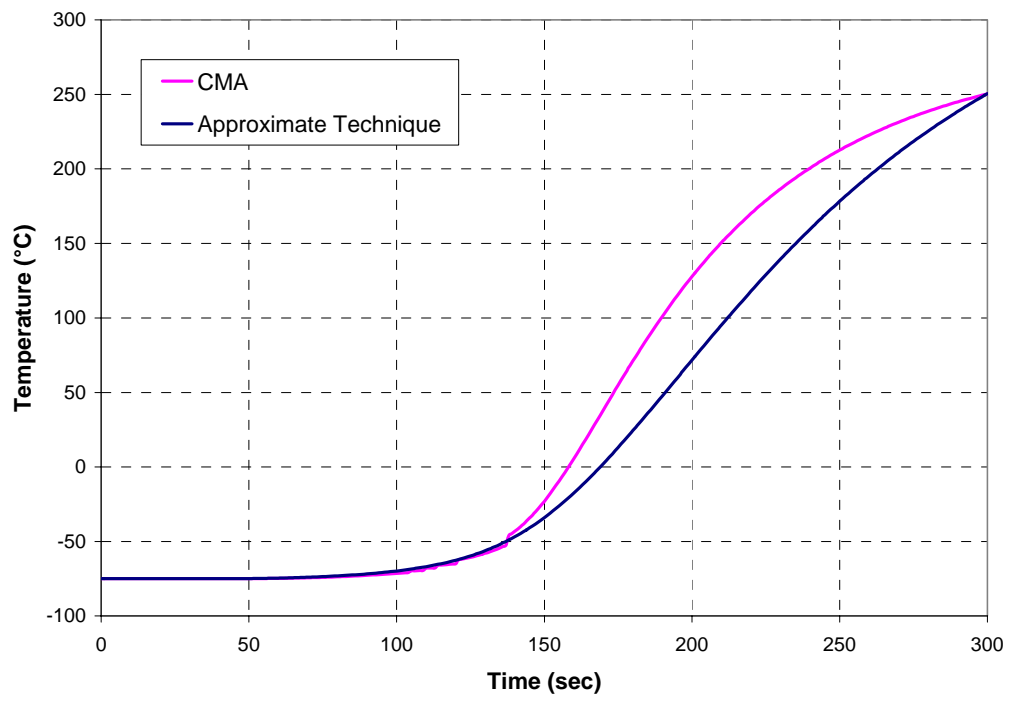

Fig. 6 Stardust bondline temperature

the bondline below $250^{\circ} \mathrm{C}$. Figure 6 shows the bondline temperature histories of the approximate and CMA solutions. The approximate and CMA solution bondline temperature profiles do not match exactly. This is primarily due to differences in thermal mass between the CMA solution and approximate solution. The TPS thickness calculated by the approximate solution is about $12 \%$ higher and therefore it has a higher thermal mass. For the same amount of energy input, a higher thermal mass system will approach the prescribed bondline temperature of $250^{\circ} \mathrm{C}$ at a slightly slower rate, as observed in Figure 6.

\section{Results and Discussion}

The approximate TPS sizing code was run assuming an Apollo capsule with a $3 \mathrm{~m}$ nose radius, ballistic coefficient of $365 \mathrm{~kg} / \mathrm{m}^{2}$, at entry velocities of $11 \mathrm{~km} / \mathrm{s}$ (lunar return) and $14 \mathrm{~km} / \mathrm{s}$ (short duration transfer Mars return). Stagnation-point thermal sizings were performed for each entry velocity and two candidate ablative material options: FM5055 Carbon Phenolic TPS and a Phenolic Impregnated Ceramic Ablator (PICA) TPS. Solutions for the 
TPS sizing were obtained using both CMA and the approximate heat of ablation, finite-difference technique. Figure 7 shows the convective and radiative stagnation-point heat rates and the combined total heat load for the $11 \mathrm{~km} / \mathrm{s}$ (lunar return) trajectory. ${ }^{16}$

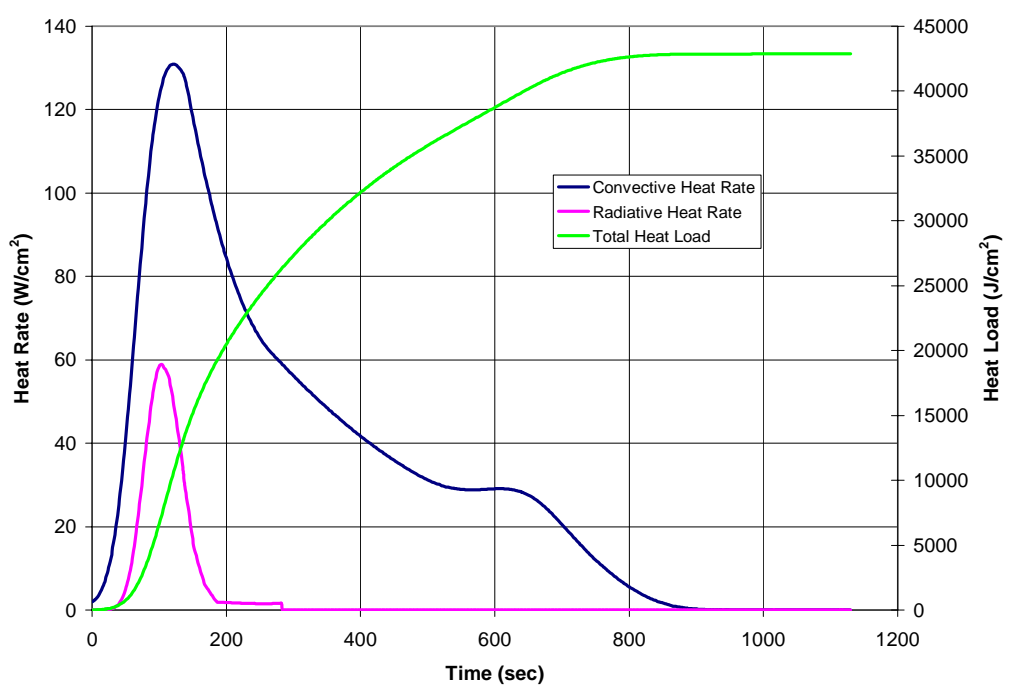

Fig. 7 Apollo capsule heat rate and heat load, 11 km/s (lunar return) entry, $3 \mathrm{~m}$ nose radius.

Table 2 shows a comparison between the CMA solution and the approximate heat of ablation, finite-difference solution. The results for the approximate solution used a recession threshold temperature of $2000 \mathrm{~K}$ for carbon phenolic and $1500 \mathrm{~K}$ for PICA. The results show very good agreement between CMA and the approximate heat of ablation, finite-difference technique. The biggest discrepancy, as anticipated, is in the recession prediction; however, the recession predictions for the approximate technique are on the conservative side with the exception of the PICA, $14 \mathrm{~km} / \mathrm{s}$ case. This $14 \mathrm{~km} / \mathrm{s}$ entry case has a significantly higher surface pressure at the time of peak heating, and

Table 2. Comparison of Stagnation-Point Total and Recession TPS Thickness

\begin{tabular}{cccccc}
\hline \hline & & \multicolumn{3}{c}{ Thickness, cm } \\
\cline { 3 - 6 } & & \multicolumn{2}{c}{$\begin{array}{c}\text { Lunar Return } \\
11 \mathrm{~km} / \mathrm{s}\end{array}$} & $\begin{array}{c}\text { Short-Transfer Mars } \\
\text { Return 14 km/s }\end{array}$ \\
\cline { 3 - 6 } Material & Thickness type & CMA & Approximate & CMA & Approximate \\
\hline \multirow{3}{*}{ FM5055 } & Total & 5.770 & 5.961 & 6.473 & 6.983 \\
& Recession & 0.005 & 0.279 & 0.574 & 1.097 \\
PICA & Total & 8.324 & 8.463 & 10.873 & 9.03 \\
\hline \hline
\end{tabular}

PICA's ablation performance is highly sensitive to pressure. These pressure effects are incorporated in the CMA solution. However, the approximate heat of ablation solution is independent of pressure. As such, it under predicts the recession. Table 3 shows the calculated thicknesses without the recession (the insulation required to maintain the bondline temperature above $250^{\circ} \mathrm{C}$ ). This is essentially a comparison of the in-depth thermal responses. Table 3

Table 3. Comparison of in-depth thermal response

\begin{tabular}{ccccccc}
\hline \hline & \multicolumn{5}{c}{ Thickness, cm } \\
\cline { 2 - 7 } & \multicolumn{5}{c}{ Apollo, 11 km/s } & Apollo, 14 km/s \\
Material & CMA & Approximate & difference & CMA & Approximate & difference \\
\hline FM5055 & 5.765 & 5.682 & 1.440 & 5.899 & 5.886 & 0.220 \\
PICA & 7.598 & 7.699 & 1.329 & 7.045 & 7.84 & 11.285 \\
\hline \hline
\end{tabular}


shows that for a high-density ablator like carbon phenolic, under these entry conditions, the in-depth thermal responses compare very well. PICA on the other hand, is a mid to low-density ablator, and under these entry conditions, a large amount of ablation and pyrolysis will occur. Looking at the PICA cases, table 3 shows the indepth thermal responses between CMA and the approximate technique do not compare as well. This is a result of the approximate solution neglecting the effects of pyrolysis energy absorption, pyrolysis gas convection through the solid and surface chemistry effects. While neglecting these physical effects, the approximate technique's in-depth solution is fairly good and is certainly within the accuracy required for conceptual design and technology assessment. Figure 8 and 9 show the surface temperature and bondline temperature histories, respectively, for the 11

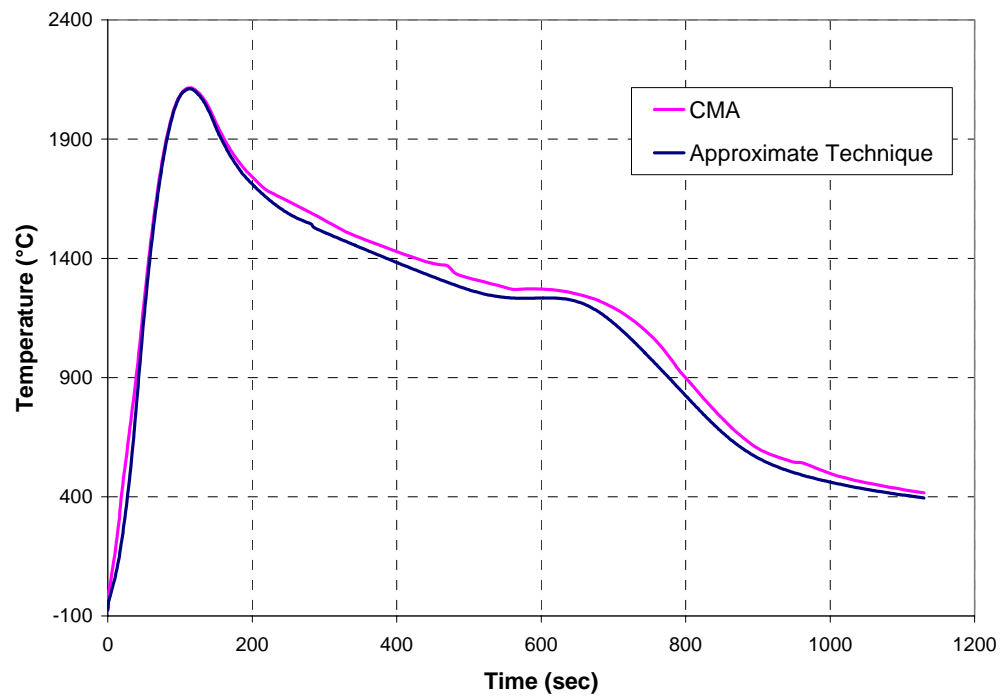

Fig. 8 Apollo type vehicle surface temperature comparison between CMA and approximate solution.

$\mathrm{km} / \mathrm{s}$ entry case for PICA. Figure 8 shows that the surface temperature matches very well up to the peak, but then begins to deviate slightly during the cool down. Figure 9 shows that the bondline temperatures match as well, and is essentially the same throughout the entire transient entry.

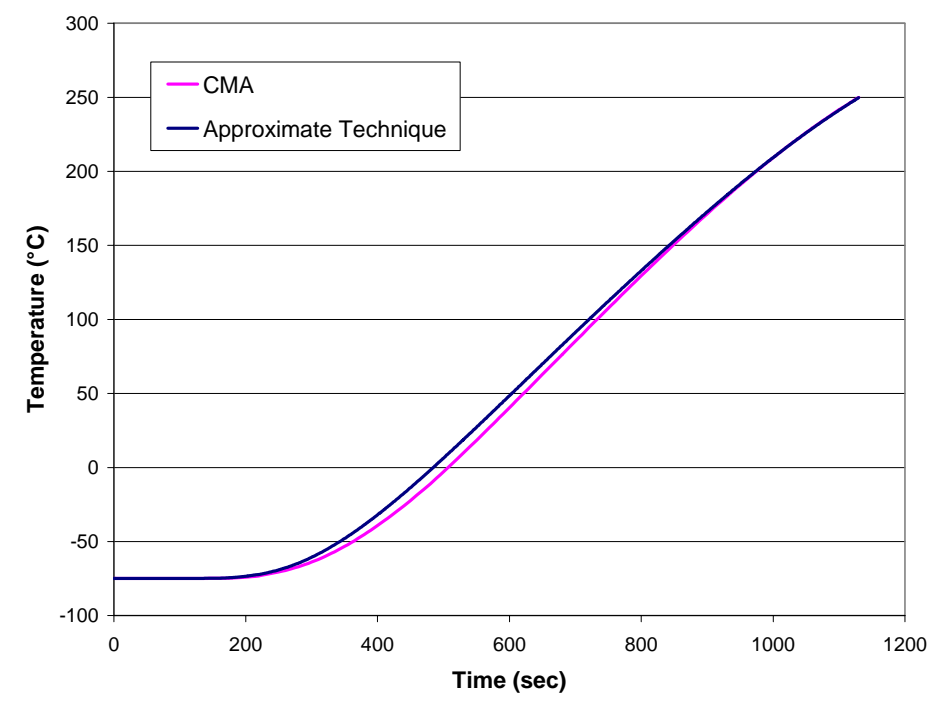

Fig. 9 Apollo type vehicle bondline temperature comparison between CMA and approximate solution. 


\section{Conclusion}

An approximate ablative TPS sizing tool has been developed for entry system design and shown to produce results that compare well to existing data for Earth and Mars entry systems. Two approaches to calculate thermal response were incorporated: (1) an industry-standard high-fidelity ablation and thermal response program, and (2) an approximate method that uses heat of ablation data to estimate heat shield recession coupled to a one-dimensional finite-difference calculation that calculates the in-depth thermal response. Engineering correlations are used to estimate stagnation-point convective and radiative heating as a function of time. This sizing tool is coupled to a trajectory simulation and can be used in an iterative loop between TPS mass and trajectory. Verification was performed by comparison to past thermal protection system sizings for the Mars Pathfinder and Stardust entry systems and new calculations were performed for an Apollo capsule entering the atmosphere at lunar and Mars return speeds. TPS sizing calculations using CMA and the approximate technique compared well with TPS sizing calculations for Mars Pathfinder and Stardust. Comparisons between the CMA thermal response and the approximate thermal response calculations were made for an Apollo capsule entering the Earth's atmosphere at 11 or $14 \mathrm{~km} / \mathrm{s}$. Results showed that for high-density materials, where little recession is expected, the approximate solution does a good job of predicting the thermal response. As the density of the material decreases and the heat rate increases, (and for the case of PICA, as surface pressure increases) the approximate solution does not perform as well. Even for this case, the approximate solution was within 11\% of the CMA predicted in-depth response, within the accuracy required for conceptual design. Including a recession threshold temperature improved the recession prediction in some cases. An enhancement to the heat of ablation method would be to make the recession calculation a function of pressure as well has heat flux. A future enhancement to the recession prediction would be to incorporate a recession prediction based on $\mathrm{B}^{\prime}$, or the non-dimensional ablation rate used in the CMA calculations.

This study also demonstrated the need to exercise caution when using an unverified CMA material response model, as in the case for the current Mars Pathfinder SLA-561V model. In this case, the approximate model was shown to be a representative sizing and could be used with confidence. Additional flight vehicles with different thermal protection system materials should also be examined; in particular a high density TPS flight vehicle should be investigated.

\section{Acknowledgements}

The authors are grateful to Bernie Laub of NASA Ames Research Center for many helpful discussions concerning material properties and ablation fundamentals. Prasun Desai of NASA Langley Research Center provided the Stardust trajectory for the verification analysis. Devin Kipp and Zachary Putnam of the Georgia Institute of Technology provided the Mars Pathfinder and Apollo trajectories for the verification and comparative analysis, respectively.

\section{References}

${ }^{1}$ Anon, “User's Manual, Aerotherm Charring Material Thermal Response and Ablation Program”, Acurex UM87-13/ATD Acurex Corporation, Aerotherm Division, Mountain View, California, November, 1987.

${ }^{2}$ Katsikas, C. J., Castle, G. K., Higgins, J. S., “Ablation Handbook Entry Materials Data and Design”, Air Force Materials Laboratory, Technical Report ARML-TR-66-262, Wright-Patterson Air Force Base, Dayton, Ohio, November 1966

${ }^{3}$ Incropera, F. P., DeWitt, D. P., Introduction to Heat Transfer, $2^{\text {nd }}$ ed., Wiley, New York, 1990, Chapter 5.

${ }^{4}$ Powell, R. W., Striepe, S. A., Desai, P. N., Braun, R. D., "Program to Optimize Simulated Trajectories (POST), Volume II, Utilization Manual”, October 1997.

${ }^{5}$ Kipp, D. M., Dec, J. A., Wells, G. A., Braun, R. D., "Development of a Planetary Entry Systems Synthesis Tool for Conceptual Entry System Design and Analysis", $3^{\text {rd }}$ International Planetary Probe Workshop, Anavyssos, Attica, Greece, June 27 - July 1, 2005.

${ }^{6}$ Sutton, K, and Graves, R. A., "A General Stagnation-Point Convective-Heating Equation for Arbitrary Gas Mixtures”, NASA TR R-376, 1971. 
${ }^{7}$ Tauber, M. E., Sutton, K., “Stagnation-Point Radiative Heating Relations for Earth and Mars Entries”, Journal of Spacecraft, Vol. 28, No 1, 1991, pp.40-42.

${ }^{8}$ Srinivasan, S., Tannehill, J. C., Weilmuenster, K. J., "Simplified curve fits for the thermodynamic properties of equilibrium air", NASA RP-1181, 1987.

${ }^{9}$ Tannehill, J. C., Anderson, D. A., Pletcher, R. H., Computational Fluid Mechanics and Heat Transfer, $2^{\text {nd }}$ ed., Taylor \& Francis, Philadelphia, 1997.

${ }^{10}$ Chapra, S. C., and Canale, R. P., Numerical Methods for Engineers, $2^{\text {nd }}$ ed., McGraw-Hill, New York, 1988.

${ }^{11}$ Spencer, D.A.; Blanchard, R.A.; Braun, R.D.; Kallemeyn, P.H.; and Thurman, S.W.; "Mars Pathfinder Entry, Descent, and Landing Reconstruction," Journal of Spacecraft \& Rockets, Vol. 36, No. 3, pp. 357-366, May-June, 1999.

${ }^{12}$ Milos, F. S., Chen, Y.-K., Congdon, W. M., Thornton, J. M., "Mars Pathfinder Entry Temperature Data, Aerothermal Heating, and Heatshield Material Response”, AIAA/ASME $7^{\text {th }}$ Joint Thermophysics and Heat Transfer Conference, Albuquerque, NM, June 15-18 1998, AIAA Paper 1998-2681.

${ }^{13}$ TPSX, Thermal Protection Systems Expert Material Property Database V4.0, NASA Ames, San Jose, CA, 2005

${ }^{14}$ Desai, P. N., Mitcheltree, R. A., Cheatwood, F. M., "Entry Dispersion Analysis for the Stardust Comet Sample Return Capsule”,Journal of Spacecraft and Rockets, Vol. 36, No. 3, 1999, pp 463-469.

${ }^{15}$ Olynick, D., Chen, Y. -K., Tauber, M. E., "Forebody TPS Sizing with Radiation and Ablation for the Stardust Sample Return Capsule”, AIAA 32 ${ }^{\text {nd }}$ Thermophysics Conference, Atlanta, GA, June 23-25, 1997, AIAA Paper 1997-2474.

${ }^{16}$ Putnam, Z.R.; Braun, R.D.; Rohrschneider, R.R.; and Dec, J.A; “Entry System Options for Human Lunar Return,” AIAA 2005-5915, AIAA Atmospheric Flight Mechanics Conference, August 2005, San Francisco, CA. 